wohl ein richtiger Kern zu Grunde liegen, und Heliopolis und sein Sonnenkult einer vorhistorischen Semiteneinwanderung in das Delta ihren Ursprung verdanken. Dieses semitische Element hätten dann die Eroberer bereits im Lande neben dem libyschen vorgefunden. Sein Vorhandensein würde auch die uralten semitischen Elemente in der ägyptischen Sprache der historischen Zeit erklären, welche hier neben einer überwiegenden Masse nichtsemitischer Bestandteile auftreten.

Wenn man somit an einzelne Annahmen und Ausführungen des Verfassers kritische Bemerkungen anknüpfen kann, so liegt das in der Natur der Sache. Was die Wissenschaft bei ihrem heutigen Stande für die ägyptische Religion beibringt, ist Stückwerk. Nur ein kleiner Teil des erhaltenen Materials ist veröffentlicht, noch viel weniger ist wissenschaftlich durchgearbeitet worden. Man kann bisher nur suchen, das Vorliegende $z u$ sichten und zu ordnen, um einer späteren zusammenfassenden Forschung möglichst die Wege zu ebnen. Das vorliegende Werk giebt eine Uebersicht über das bereits Geleistete und ergänzt es durch Einzelnotizen und Abschnitte, welche in den bisherigen Darstellungen der ägyptischen Religion keinen Platz zu finden pflegten. Der Verfasser beginnt seine Darstellung mit einer Reihe von allgemeinen Ausführungen über die ägyptischen Götter, den Tierkult, die Göttlichkeit der Könige, den Begriff der Gottheit, die Götterkreise, die henotheistischen Vorstellungen. Dann folgt eine Schilderung der Unterwelt, ibrer Dämonen und der Verdammten, eine Besprechung der Schöpfungsmythen, des Sonnengottes Rā und der an ihn anknüpfenden Gottessagen, des Gottes Thoth und seines Kreises, der Hathoren, der Horus-Götter und der Triade von Memphis. Der zweite Band erörtert zunächst die Triade von Theben, den Nilgott, die Triade von Elephantine, den durch Amenophis IV. zum Hauptgotte des Landes erhobenen Aten, den Götterkreis von Heliopolis, dann in ausführlicher Darstellung Osiris und die Götter seines Kreises, und in kürzerer Form die fremden Götter im Nilthale. Dann folgen in tabellarischer Anordnung Gestalten, welche Budge als Miscellaneous Gods zusammenfasst, wie die Gottheiten der Elle, der Tage, Monate, Stunde, Winde, Sternbilder und Einzelsterne, des Totenbuches u. s. w. Es sind dies sehr bequeme Uebersichten (ähnliche Listen finden sich auch sonst in dem Werke ver- teilt, wie die Götter der Pyramidentexte S. $79 \mathrm{ff}$., die Nomosgötter S. $96 \mathrm{ff}$. u. a. m.), welche in ähnlicher Vollständigkeit in ägyptischen Religionsgeschichten bisher nicht dargeboten worden sind. An sie schliesst sich eine Behandlung der heiligen Tiere. Ein eingehendes Register bildet den Schluss.

Den Text begleiten zahlreiche Farbendrucktafeln und Clichés mit Bildern der einzelnen Gottheiten und verschiedenartigen sonstigen mythologischen Vorstellungen, wie Räumen der Unterwelt, der einzelnen Phasen der Auferstehung des Osiris u. a. m. Als Ganzes bildet das Werk eine fleissige und übersichtliche Zusammenstellung der für die ägyptische Religion aus dem Altertume vorliegenden Angaben und reiht sich als brauchbares und reichhaltiges Handbuch den zahlreichen früheren Schriften des Verfassers über ägyptische religiöse Texte und Fragen ergänzend und weiterführend an.

Bonn.

\section{Un peu de lumière!}

Dans sa récension bienveillante de mes Études érangéliques (0. L. Z. 1904, Col. 21-22), M. J. Bergmann discute les deux interprétations proposées par M. Chajes et moi pour le passage Marc IX, 49 a ainsi

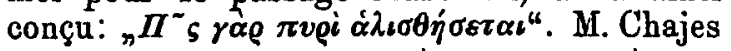

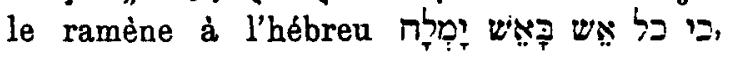
tandis que moi, je suppose qu'au lieu de $\pi v \rho i$ il faut lire un mot qui signifie ăoros ou бiros. M. Bergmann remarque: "Halévy ahnt das Richtige et et pense que l'original por-

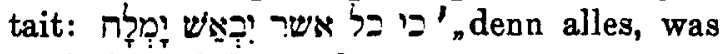
verfault, wird gesalzen"; שא facilement devenir mon hypothèse, parce que je crois que l'original de Marc était rédigé en araméen; mais je reconnais que la leçon de M. Bergmann doit étre sérieusement examinée.

M. S. Krauss (ibidem, Col. 111) n'aime pas les conjectures, et en premier liea „die von Halévy, der angeblich das Richtige ahnt." Je me dis: M. Krauss nous fournira certainement quelque chose de positif, d'indubitable. Mon attente est renforcée par la periode assurée qui suit:

"Ich glaube diesen vagen Vermutungen einen Riegel vorschieben zu müssen, durch Hinweis auf zwei Veröffentlichungen, die jene alte crux interpretum für immer beseitigen, die aber, weil abseits gelegen, jenen Herren unbekannt geblieben zu sein scheinen." 
De ma part, je me consolerai de ma médiocre érudition bibliographique, mais la perspective de me heurter contre une porte verrouillée, ne me va pas du tout.

$\mathrm{Je}$ lis donc fébrilement la suite. Je trouve $1^{0}$ que Kirsop Lake a fait la découverte que primitivement il n'y avait pas dans Marc. IX, 49b KAITACA $\Theta Y C I A$ ANIC mais KAI TIACAOYCIA ANA^WOHETAI „et toute substance est anéantie"; $2^{0}$ que cette leçon est confirmée par un codex latin d'Afrique, ainsi que par un codex grec d'une Lavra sur le mont Athos; $3^{0}$ que Ricci, qui accepte cette lecture, a tort d'attribuer la leçon courante à un juif converti; $4^{0}$ que la "Revised Bible ${ }^{\alpha}$ (Oxford 1899) omet cette seconde phrase et en prend seulement note sur la marge.

Ce sont des choses bonnes à savoir, mais où est Marc. IX, 49a TACIAPTIYPIANICOHETAI qui est l'objet de nos hypothèses? Je le cherche sans le trouver. Dans ma déroute je demande un peu de lumière!

La conclusion de $M$. Krauss ne m'éclaire pas davantage. Je lis:

"An unserer Stelle ist also nicht von Salz die Rede. Bloss Vers 50 enthält ein Logion Jesu, welches vom Salz ausgeht; dieses Logion entspricht dem, was wir im Matt. 5, 13 und Luc. 14, 34 lesen. Jene Korruptele also, die in Vers 49 das Salz hineinschmuggelte, wollte zu Vers 50 eine Brücke bilden; wie wir sehen ganz fehlerhaft. "

Mais enfin toutes ces corruptions apparentes ou vraies ne concernent que la seconde partie du verset 49 et que fait on de la première partie qui se troure intact dans tous les codices occidentaux et orientaux? $\mathrm{Je}$ suis donc obligé de demander encore un peu de lumière!

En attendant, une pensée me tourmente. Je crains que l'effort dépensé pour nous verrouiller la porte, n'ait joué à notre critique le tour que les talmudistes expriment par la formule:

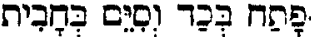

$$
\text { J. Halévy. }
$$

Ein neues Datum zur Geschichte Assyriens. Von F. E. Peiser.

In der No. 21 der Mitteilungen der Deutschen Orient-Gesellschaft teilt Delitzsch im Anschluss an die Berichte Andrä's ${ }^{1}$ ) die

1) Dass Herr Andrä sich bemüht, Assyrisch lesen zu lernen, ist awerkennenswert. Dass dadurch die dringende Forderung naoh Assyriologen an den Ausgrabungsstatten abgeschw\&cht werden könnte, wird deshalb nicht zugestanden werden können. chronologischen Angaben der Steintafelinschrift des Königs Salmanassar I. mit.

Danach hat Samši-Adad, der Priester Ašur's, 580 Jahre vor Salmanassar I. regiert, und Erišu, der Priester Ašur's, 159 Jahre vor jenem. Mit der von Scheil Recueil 1900 mitgeteilten Inschrift von Kalah Schergat, siehe OLZ. III 476, stellt Delitzsch die Liste her:

Ušpia (Vor. A-ušpia)

Ilušûuma, dessen Sohn

Erišum, dessen Sohn

Ikûnum

Išme-Dagan, dessen Sohn

Śamši-Adad.

Mit Recht macht Delitzsch darauf aufmerksam, dass durch die neue chronologische Angabe die ïbliche Ansetzung Tiglatpileser's I. auf $1120 \mathrm{zu}$ revidieren ist.

Da Tukulti-Ninib nach Sanherib's Angabe $600+690=1290$ zu setzen ist, sein Vater Salmanassar also um rund 1320 gelebt haben wird, so kommt Samši-Adad auf 1900. Nach den Angaben Tiglatpileser's regierte nun Ašur-dân 641 Jahre nach SamšiAdad, also um 1260. Ašur-dân war Zeitgenosse des Zamama-šum-iddin von Babylon, dessen Datum auf +1265 durch die Argabe Nabonids bestimmt ist $(800+540=1340$, $1340-75=1265$ ). Da nun von Ašur-dân bis Tiglatpileser nach dessen eigener Angabe 60 Jahre zu rechnen sind, so kommt TP. I. auf rund 1200, oder, da für die Regierung Ašur-dân's, als eines langlebigen Herrschers, ein grösserer Spielraum angenommen werden darf, auf ca. 1180. Auf die gleiche Zahl führt die Angabe der Königsliste; denn wenn Zamamašumiddin um 1265 zu setzen ist, so beginnt die Paše-Dynastie um 1262 und schliesst, nach meiner Korrektur der Gesamtzahl, um 1130. Die 4 Schlussregierungen dauerten zusammen 45 Jahre, dazu einige Jahre für Marduk-šāpik-kullatgerechnet, giebt die Möglichkeit, Marduk-nâdin-ahi, den Gegner T. P. I', auch auf $1180 \mathrm{zu}$ setzen.

Damit würde nun also wirklich die Sanherib-Bavian-Angabe als um rund 60 Jahre zu niedrig erwiesen sein und somit die Vermutung Rost's MVAG. 1897. II S. 16 eine hübsche Bestätigung erfahren.

\section{Der Orientalistenkongress in Algier.}

Der 14. Kongress soll im April 1905 in Algier stattfinden; sehr weise ist diesmal von 